\title{
BARRERAS EN EL ACCESO A LA DIRECCIÓN ESCOLAR: DIFERENCIAS ENTRE PROFESORAS Y DIRECTORAS DE CENTROS DE EDUCACIÓN INFANTIL Y PRIMARIA*
}

\author{
BARRIERS IN THE ACCESS TO SCHOOL MANAGEMENT: DIFFERENCES \\ BETWEEN TEACHERS AND HEAD-TEACHERS FROM EARLY CHILDHOOD \\ AND ELEMENTARY EDUCATION SCHOOLS
}

\author{
M. Teresa Padilla Carmona** \\ Universidad de Sevilla
}

\section{RESUMEN}

Se presenta una investigación que indaga en las barreras que limitan el desarrollo profesional de las docentes. Así, se han analizado los obstáculos que condicionan el acceso a la dirección escolar en la enseñanza infantil y primaria. Mediante un muestreo polietápico se ha seleccionado a 622 profesoras y 206 directoras en Andalucía y se les aplicó una escala sobre Condicionantes en el acceso a la dirección, cuyas dimensiones reflejan barreras en el desarrollo profesional. Los resultados muestran que, tanto directoras como maestras, perciben el conflicto de roles y la falta de políticas de apoyo a la maternidad como las barreras que más les afectan.

Palabras clave: barreras en el desarrollo profesional femenino; acceso a la dirección escolar; diferencias profesoras-directoras; estudio tipo encuesta.

\section{ABSTRACT}

The article analyzes the barriers that hinder women access to school management in early and elementary education. Through a several-stages method, a sample of 622 female teachers and 206 headteachers from Andalusia was selected. Data were collected through a scale named Barriers in the ac-

\footnotetext{
* Este artículo es un trabajo parcial de la investigación La mujer en la dirección de los centros escolares: Análisis de las barreras que dificultan el acceso a la dirección y de los procesos de gestión y liderazgo escolar, financiada por el Ministerio de Ciencia y Tecnología dentro del Plan Nacional de I+D+I (BOE de 15 enero de 2002).

** Dra. en Pedagogía. Profesora Titular del Dto. de Métodos de Investigación y Diagnóstico en Educación de la Universidad de Sevilla (Facultad de Ciencias de la Educación).

Líneas de investigación: desarrollo profesional, desarrollo profesional femenino, análisis del sexismo en el ámbito educativo, evaluación del aprendizaje en la Universidad. Correo electrónico: tpadilla@us.es
} 
cess to the school management, whose dimensions reflect possible barriers to career development. Results show that role-conflict and the lack of motherhood-supporting politics are the most important barriers for both teachers and head-teachers. Also, head-teachers responses show higher self-esteem and social skills than teachers'.

Key words: barriers to the career development of women; access to school management; teachers/ head-teachers differences; survey.

\section{Introducción}

Para comprender y explicar las diferencias propias del proceso de desarrollo profesional femenino, gran número de autoras/es usan el término barreras profesionales (e.g., Fitzgerald y Weitzman, 1992; McWhirter, Torres y Rasheed, 1998; Russell y Burgess, 1998) para referirse a "eventos o condiciones, tanto internas de la persona, como de su ambiente, que dificultan el progreso profesional" (Swanson y Woitke, 1997: 446). Esta definición no sólo nos ofrece una aproximación al concepto de barreras, sino que también nos aporta una primera clasificación de las mismas (barreras internas y externas).

Las barreras externas son caracterizadas como específicas del entorno laboral e incluyen la discriminación en los procesos de selección de personal, en la promoción y evaluación del desempeño laboral, así como la exclusión de la mujer de ciertos puestos, las diferencias salariales respecto a sus compañeros varones, la falta de políticas adecuadas para atender la maternidad, e incluso el acoso sexual en el trabajo (Gimeno y Rocabert, 1998; Padilla, 2001; Sánchez García, 2004).

Otros obstáculos externos en el acceso y desarrollo de un empleo que pueden encontrar las mujeres son: la falta de mentoras y ejemplos femeninos a los que imitar y cuya influencia seguir, la dificultad para desarrollar en su ambiente laboral una fuerza política (es decir, una red de relaciones y contactos que le confiera cierto poder dentro de la organización), la falta de apoyo por parte de la pareja y las amistades, encontrando a su alrededor un ambiente desfavorable. En los recientes trabajos de Suárez Ortega (2004 y 2008) se evidencia que la propia pareja y los/as hijos/as pueden ejercer una influencia negativa en el proceso de re-incorporación de la mujer adulta al mercado laboral.

Por su parte las barreras internas hacen referencia a conflictos internos y presiones psicológicas que experimenta la mujer y que conllevan su autoexclusión de determinadas esferas del entorno laboral. Algunas de estas barreras internas son la baja autoestima, la falta de expectativas de éxito, el conflicto entre el rol de cuidadora del hogar y el de trabajadora (Gimeno y Rocabert, 1998; Padilla, 2001). En general, los condicionantes externos van ejerciendo una presión sobre las expectativas de las mujeres, de forma que sus bajas expectativas de éxito se constituyen en una nueva barrera, en tanto que reducen la probabilidad de que ésta ponga en prácticas comportamientos que van a permitir superar positivamente los obstáculos.

En esta línea, una barrera que ha recibido especial atención es el conflicto entre los roles de trabajadora y cuidadora del hogar. Gran parte de las barreras anteriormente mencionadas se relacionan o dependen de esta relación entre el mundo privado del hogar y el mundo público del trabajo, relación que suele ser compleja y no admite planteamientos simplistas. Por un lado, por cuestiones culturales y educativas, a las mujeres se les enseña a valorar y a tener entre sus prioridades el matrimonio y la maternidad, relegando 
de esta forma, el trabajo a un segundo plano. Por esta razón, es muy habitual que se produzca lo que se denomina "sobrecarga de roles". La mujer asume sus responsabilidades en el terreno laboral al tiempo que mantiene las relativas al rol de madre, esposa y ama de casa. Esto se traduce en un conflicto entre las demandas de ambos roles vitales que acaba sobrecargando a la mujer y teniendo consecuencias negativas en su desarrollo profesional y personal, y más concretamente, en sus niveles de satisfacción con el trabajo y su vida.

El ámbito educativo es un sector ocupacional en el que tradicionalmente ha habido una alta presencia femenina. Por ello, y dado que nuestro estudio se desarrolla en dicho ámbito, se plantea como necesario profundizar en la situación de las mujeres docentes.

En general, la enseñanza es una profesión con gran presencia de mujeres. Sin embargo, la presencia femenina y masculina es muy desigual según los diferentes niveles, materias o áreas profesionales y, también según los puestos de responsabilidad. En el trabajo de $\mathrm{Mu}-$ ñoz-Repiso (2003), se pone de manifiesto que en el contexto europeo, mientras que alrededor del $75 \%$ del profesorado en enseñanza primaria son mujeres (llegando al $95 \%$ en el caso de infantil), tan sólo un 50\% de ellas desempeña puestos de dirección. En nuestro país las cifras son similares: según el estudio de Grañeras (2003), mientras que casi el 63\% del profesorado de infantil y primaria son mujeres, sólo un $42,21 \%$ de las docentes ejercen como directoras. En la C.A. Andaluza, durante el curso 2003/04, en primaria e infantil había un $65,6 \%$ de profesoras y sólo un 33,6\% de estas docentes ejercían la dirección escolar. En cursos anteriores (concretamente, en 2000/01), estas cifras era aún más desiguales: un $29,13 \%$ de directoras frente $70,78 \%$ varones directores.

A ello se añade que no sólo es más frecuente encontrar hombres en las tareas de dirección, sino que también se encuentran con más frecuencia en centros más grandes y con mayor número de alumnado y profesorado. Como sugiere el estudio de Díez Gutiérrez (2004), el tamaño de los centros parece estar en relación con la imagen social de poder que se tiene de ellos, con la dificultad o envergadura de la tarea a desempeñar y con el nivel de lucha y competencia por el acceso y ejercicio del poder en los mismos.

Por tanto, la superioridad femenina en cuanto a número no está equiparada en las organizaciones educativas con su presencia en los puestos de responsabilidad y de poder (Bardisa 1995). Al ser usual que en los centros de Infantil y Primaria sea un varón el responsable de la dirección y la mayoría del profesorado mujeres, se da lo que en el trabajo de Ball (1994) se denomina como el "harén pedagógico". Pese a ello, parece ser que cuando los puestos de responsabilidad son ocupados por mujeres se dan procesos altamente positivos para el funcionamiento de la organización (Carrasco, 2002; Coronel, Moreno y Padilla, 2002).

\section{Método}

Se ha utilizado el método de encuesta cuyas características son la formulación de preguntas y la pretensión de generalizar los resultados a la población, rasgos que justifican su elección en nuestro estudio. Se trata, por tanto, de un estudio descriptivo que finalizó en octubre de 2005 y que se ha desarrollado en las siguientes fases: 1) revisión de las principales aportaciones en torno a las barreras en el desarrollo profesional femenino y selección de las dimensiones del estudio; 2) construcción de la primera versión de la escala-cuestionario 
y validación de su contenido por personas expertas (Padilla, 2005) para la elaboración de la versión definitiva; 3) selección de las muestras de directoras y profesoras participantes; 4) recogida de datos; $y$, finalmente, 5) análisis de los mismos y obtención de resultados.

\section{Objetivos}

El objetivo principal de la investigación desarrollada consistió en identificar las barreras y condicionantes que limitan el acceso a la dirección escolar por parte de las docentes en la enseñanza infantil y primaria. Concretamente, aquí nos centramos en:

1. Conocer cuáles son los obstáculos externos que limitan y condicionan el acceso de las profesoras a la dirección de centros, así como las presiones psicológicas o conflictos internos que llevan a que rehúsen desempeñar puestos de responsabilidad en la administración escolar. Nos interesa recabar esta información tanto desde la perspectiva de las maestras, como desde la de las directoras de centros escolares.

2. Identificar las principales diferencias en las percepciones que ambos grupos tienen respecto a las barreras y obstáculos en su desarrollo profesional.

\section{Población y muestra}

Las poblaciones del estudio estaban compuestas, respectivamente, por el total de las profesoras y las directoras de los centros de educación infantil y primaria de Andalucía. Para la selección muestral se siguió un procedimiento polietápico que combinó un muestreo aleatorio estratificado, para la selección de las docentes y directoras por cada provincia andaluza, con un muestreo por conglemerados, en el caso de las profesoras (es decir, seleccionando a todas las profesoras de los centros que resultaron elegidos). El tamaño final de la muestra y su distribución respecto a las provincias andaluzas se recoge en la tabla 1 .

\section{Técnica de recogida de datos}

Se diseñó un cuestionario ad-hoc con dos versiones (para docentes y directoras), que contiene una escala de 36 ítems denominada Escala de opiniones sobre los condicionantes en el acceso a la dirección ${ }^{1}$. El instrumento contiene además otros dos bloques de preguntas que recaban información de carácter profesional y vital sobre las encuestadas junto con sus opiniones y experiencias ante la dirección escolar. En lo que se refiere a la escala, esta se compone de siete dimensiones que reflejan las barreras que las docentes pueden experimentar a la hora de optar por el desempeño de un cargo directivo: conflicto de roles, autoestima, expectativas de éxito, políticas de ayuda a la maternidad, acceso a la información, actitudes hacia la gestión femenina y asertividad.

1. En la versión del instrumento aplicada en el grupo de directoras, la escala tiene 44 ítems ya que incluye cuatro dimensiones relativas a las ventajas e inconvenientes, tanto personales como profesionales, que atribuyen al desempeño de la dirección. Esta información corresponde a otros objetivos de investigación. 
TABLA 1. Composición de la muestra por provincia.

\begin{tabular}{|l|c|c|c|c|}
\hline \multirow{2}{*}{} & \multicolumn{2}{|c|}{ DIRECTORAS } & \multicolumn{2}{c|}{ PROFESORAS } \\
\cline { 2 - 5 } & $\begin{array}{c}\text { Porcentaje de } \\
\text { contribución }\end{array}$ & $\begin{array}{c}\mathrm{N}^{\circ} \text { de directoras } \\
\text { incluidas }\end{array}$ & $\begin{array}{c}\text { Porcentaje de } \\
\text { contribución }\end{array}$ & $\begin{array}{c}\mathrm{N}^{0} \text { de profesoras } \\
\text { incluidas }\end{array}$ \\
\hline Almería & 14.56 & 30 & 9.97 & 62 \\
\hline Cádiz & 5.83 & 12 & 11.73 & 73 \\
\hline Córdoba & 11.65 & 24 & 10.13 & 63 \\
\hline Granada & 15.53 & 32 & 5.15 & 32 \\
\hline Huelva & 7.28 & 15 & 13.02 & 81 \\
\hline Jaén & 13.11 & 27 & 10.29 & 64 \\
\hline Málaga & 14.10 & 29 & 9.16 & 57 \\
\hline Sevilla & 17.96 & 37 & 30.55 & 190 \\
\hline Total en C.A. Andal. & - & 206 & - & 622 \\
\hline
\end{tabular}

A fin de evitar la aquiescencia, los ítems fueron redactados tanto en sentido afirmativo como negativo. Asimismo, las respuestas oscilan entre 1 (mínimo nivel de acuerdo con el enunciado) y 6 (máximo acuerdo). Se trata, por tanto, de un número par de rangos de respuestas que tiene por objeto evitar, en lo posible, la tendencia central.

\section{Procedimientos utilizados en el análisis de datos}

Para el análisis de la información, se han estimado los estadísticos descriptivos de tendencia central (concretamente, la media) y de dispersión (desviación típica) para los diferentes ítems y dimensiones de la escala. Asimismo, se ha aplicado la prueba $\mathrm{T}$ de contraste de medias para identificar diferencias de opinión entre directoras y profesoras, utilizando un nivel de confianza del 99\% para la decisión estadística.

\section{Resultados}

En la tabla 2 se exponen los resultados obtenidos por los dos grupos objeto de análisis (docentes y directoras) en lo relativo a las barreras y condicionantes que perciben en el acceso y desempeño de la dirección escolar. En dicha tabla también se muestran los resultados de las contrastes de medias que han resultado significativos. 
TABLA 2. Condicionantes en el acceso a la dirección

\begin{tabular}{|c|c|c|c|}
\hline & $\begin{array}{c}\text { Profesoras } \\
\mu / \sigma\end{array}$ & $\begin{array}{c}\text { Directoras } \\
\mu / \sigma\end{array}$ & $T(p)$ \\
\hline Conflicto de roles & $2,77(1,50)$ & $2,70(1,30)$ & \\
\hline (+) Ascender aunque reste tiempo & $2,25(1,34)$ & $1,73(1,29)$ & $4,79(0,000)$ \\
\hline (-) Cuidar hogar resta tiempo & $4,22(1,68)$ & $4,80(1,58)$ & $-4,30(0,000)$ \\
\hline (+) Ascender más importante & $1,87(1,18)$ & $1,58(1,03)$ & $3,23(0,001)$ \\
\hline Autoestima & $3,63(1,52)$ & $4,80(1,35)$ & $-13,69(0,000)$ \\
\hline (-) Dudo de mi capacidad & $3,84(1,60)$ & $4,99(1,56)$ & $-8,90(0,000)$ \\
\hline (+) Me considero capacitada & $3,67(1,56)$ & $5,07(1,04)$ & $-14,31(0,000)$ \\
\hline (-) Carezco de autoridad & $3,90(1,57)$ & $4,68(1,62)$ & $-6,04(0,000)$ \\
\hline (+) Aptitudes y cualidades útiles & $3,11(1,34)$ & $4,44(1,16)$ & $-12,54(0,000)$ \\
\hline Actitudes hacia la gestión femenina & $4,44(1,52)$ & $4,77(1,50)$ & $-3,56(0,000)$ \\
\hline (+) Respeto de padres y madres & $4,27(1,50)$ & $4,52(1,52)$ & \\
\hline (+) Respeto de alumnos/as & $4,20(1,58)$ & $4,93(1,40)$ & $-6,20(0,000)$ \\
\hline (-) Burlas compañeros & $4,85(1,49)$ & $4,82(1,57)$ & \\
\hline Políticas de apoyo a la maternidad & $2,43(1,51)$ & $2,56(1,60)$ & \\
\hline (+) Las políticas son suficientes & $2,85(1,66)$ & $3,14(1,76)$ & \\
\hline (+) Existen ayudas económicas & $2,10(1,28)$ & $1,81(1,28)$ & \\
\hline (-) Las políticas deben mejorar & $2,51(1,60)$ & $2,81(1,75)$ & $2,67(0,008)$ \\
\hline Acceso a la información & $4,48(1,49)$ & $4,47(1,66)$ & \\
\hline (+) Las docentes disponen de información & $3,83(1,56)$ & $3,64(1,85)$ & \\
\hline (-) Los docentes más informados para planificar & $5,02(1,36)$ & $5,12(1,47)$ & \\
\hline (-) Los docentes acceden a más información & $4,60(1,55)$ & $4,67(1,66)$ & \\
\hline Asertividad en las relaciones & $3,65(1,43)$ & $3,93(1,45)$ & $-5,08(0,000)$ \\
\hline (-) Evito hacer preguntas & $4,52(1,61)$ & $4,96(1,57)$ & $-3,35(0,001)$ \\
\hline (-) Incómoda al negar petición & $3,20(1,59)$ & $3,59(1,48)$ & \\
\hline (-) Guardar mis opiniones & $4,01(1,53)$ & $5,35(1,10)$ & $-2,90(0,000)$ \\
\hline (+) Afronto situaciones de comunicar malas noticias & $3,01(1,42)$ & $2,51(1,48)$ & $-4,96(0,000)$ \\
\hline (+) Expreso mi enfado ante compañeros & & & $-2,78(0,006)$ \\
\hline (-) A veces no se que decir & $3,42(1,38)$ & $3,58(1,66)$ & \\
\hline (+) Fácil relación con los compañeros & $4,31(1,45)$ & $4,30(1,29)$ & $-3,70(0,000)$ \\
\hline (+) Alabo a mis colegas por su buen trabajo & $5,00(1,20)$ & $3,30(1,62)$ & $-7,16(0,000)$ \\
\hline (+) Si he de negarme lo hago con facilidad & $5,05(1,06)$ & $3,77(1,56)$ & \\
\hline (-) Aunque tenga prisa me cuesta cortar & $2,92(1,41)$ & $5,57(0,82)$ & \\
\hline (-) Me cuesta recordar trabajos compañeros & $2,99(1,48)$ & $3,86(1,56)$ & $3,95(0,000)$ \\
\hline (-) Prefiero ceder para evitar problemas & $3,40(1,47)$ & $2,86(1,32)$ & $-3,68(0,000)$ \\
\hline (+) Pido favores & $3,27(1,50)$ & $4,37(1,54)$ & \\
\hline (-) Difícil pedir que hagan cosas & $3,15(1,46)$ & $4,60(1,73)$ & $-3,21(0,001)$ \\
\hline (+) Expreso mis sentimientos & $2,82(1,48)$ & $3,04(1,42)$ & \\
\hline \multirow[t]{2}{*}{ (+) Hablo si estoy descontenta con trabajo } & $3,82(1,47)$ & $3,52(1,58)$ & $-3,51(0,000)$ \\
\hline & $3,57(1,36)$ & $4,23(1,40)$ & $-6,66(0,000)$ \\
\hline
\end{tabular}


Empezando por el grupo de profesoras, de forma general, puede destacarse que las dimensiones que más acuerdo suscitan con el contenido de los ítems — una vez transformados los valores correspondientes de los ítems negativos- son acceso a la información (promedio de $4,48^{2}$ ) y actitudes hacia la gestión femenina $(4,44)$. Tal y como están expresados los polos positivos y negativos de las distintas dimensiones, estas serían las supuestas barreras que menos inciden en el acceso de las docentes a los puestos directivos.

Las barreras a las que otorgan mayor incidencia en su desarrollo profesional son: politicas de apoyo a la maternidad $(2,43)$ y conflicto de roles $(2,77)$. Aunque con contenido diferente, en cierto modo, ambas reflejan que la dedicación de la mujer a la familia tiene un gran peso a la hora de plantearse un ascenso. Esto no implica que las docentes consideren el espacio familiar como una barrera impuesta desde el punto de visto social, como sí lo hacen con la insuficiencia de políticas que apoyen la maternidad. Si nos atenemos al contenido de los ítems en la dimensión conflicto de roles, más bien se trata de una barrera "auto-impuesta": el ascenso es algo no deseado si implica una re-estructuración de sus tiempos familiares, conllevando opciones y prioridades vitales.

Asimismo, conviene destacar que las dos dimensiones restantes (asertividad $-3,65-\mathrm{y}$ autoestima $(3,63)$, obtienen valores intermedios, lo que quizá indique que son factores con una importancia relativa en comparación con los demás. Ambos tienen un contenido hasta cierto punto común, ya que se refieren a considerarse una persona capaz de ejercer la dirección y relacionarse asertivamente con otras personas del entorno. Por tanto, podemos decir que las docentes no manifiestan tener un nivel alto de autoestima ni una alta valoración de sus habilidades sociales y relacionadas con la asertividad.

En el grupo de directoras, por otra parte, también se percibe que la dimensión conflicto de roles $(2,70)$ constituye una barrera que afecta en cierta medida al desempeño de la dirección. En principio, las directoras no están muy de acuerdo con que cuidar de la familia les reste tiempo para dedicarse a su profesión, pero consideran que ascender en su trabajo no debe ser más importante que su dedicación a la familia.

En relación con la dimensión autoestima, el promedio puede considerarse alto $(4,80$ sobre 6), lo que implica que las directoras no tienden a dudar de su capacidad para ejercer la dirección, sino que, todo lo contrario, se consideran con las cualidades y aptitudes necesarias para el cargo. En cuanto a las actitudes hacia la gestión femenina, las encuestadas opinan que ni las familias ni los alumnos y alumnas tienden a comportarse de forma diferente con un director varón a como lo harían con ellas. Sin embargo, las opiniones sobre las políticas de apoyo a la maternidad reflejan que los recursos y ayudas existentes en la actualidad no son suficientes y, por tanto, podrían condicionar el acceso a la dirección por parte de las mujeres. Asimismo, las respuestas a los ítems de la dimensión acceso a la información no reflejan que ellas crean que los docentes varones suelan manejar más información sobre oportunidades de ascenso y, en consecuencia, planifiquen mejor su carrera profesional.

En la dimensión relativa al nivel de asertividad que poseen las directoras, el promedio global obtenido es de 3,93, lo que puede considerarse como un valor medio-alto. Las directoras, por tanto, denotan una cierta asertividad, en sus distintos matices, aunque quizá este comportamiento asertivo debiera alcanzar valores más altos, dadas las características de su

2. Los valores pueden oscilar entre 1 (mínimo) y 6 (máximo). 
cargo. El análisis del contenido de los ítems con medias más altas y más bajas, evidencia que directoras no muestran grandes dificultades para las relaciones y los halagos positivos, pero sí a la hora de hacer peticiones y reclamaciones.

Pasando ya a la comparación entre los dos grupos, y teniendo en cuenta los promedios globales obtenidos en cada dimensión, las diferencias más grandes -y las únicas estadísticamente significativas-, en cuanto a medias globales de las dimensiones se encuentran en las dimensiones autoestima (1.17), actitudes hacia la gestión femenina (0.33) y asertividad (0.28).

Se aprecia una importante diferencia en autoestima a favor de las directoras, las cuales tienden a mostrar una imagen más positiva de sí mismas. Tiene también este colectivo un mayor reconocimiento de sus habilidades asertivas y para las relaciones. Por otra parte, ellas parecen considerar como menos sexistas y paralizantes las actitudes ante la gestión femenina, ya que también han puntuado más alto en esta dimensión.

Conviene destacar la única diferencia de medias, a nivel de las dimensiones, entre profesoras y directoras que se da en sentido inverso - aunque de forma no significativaal que venimos comentando; es decir, el único caso en que la media de las profesoras supera a las de sus colegas. Esto sucede en la dimensión conflicto de roles, en la que se aprecia que las maestras tienden a mostrarse más de acuerdo con la idea de que la dedicación al ámbito familiar no les reste tiempo en el terreno profesional, especialmente de cara al ascenso. En cualquier caso, estamos ante valores bajos en ambos colectivos, es decir, que tanto unas como otras no desean ascender si eso implica una menor dedicación a la familia.

Sin embargo, entrando en el análisis de las diferencias a nivel de los ítems, se observa que estas son significativas desde el punto de vista estadístico. En el caso del ítem cuidar del hogar resta tiempo..., son las directoras las más sensibles a este hecho, mientras que las docentes obtienen medias más altas en cuanto a su acuerdo con que el ascenso profesional se traduzca en una menor dedicación a la familia. Es curiosa, por tanto, esta diferencia a favor del colectivo que, supuestamente, no ha ascendido profesionalmente al cargo de directora de centro.

En el resto de ítems se observa la tendencia a que sean las directoras las que obtengan promedios más altos, aunque en algunos casos las diferencias son muy pequeñas y no significativas. Esto implica, en general, una menor incidencia de la barrera o condicionante en cuestión: bien porque tienen más autoestima o asertividad, bien porque piensan que las actitudes hacia la gestión femenina son poco sexistas, etc.

En el nivel de los ítems, las diferencias más altas se concentran, como cabe esperar, en los ítems correspondientes a autoestima, donde las directoras sobrepasan en un punto la media de sus colegas (como dudo de mi capacidad..., me considero capacitada..., reúno las aptitudes y cualidades...), siendo significativas estas diferencias de medias. Sin embargo, ítems de otras dimensiones también muestran diferencias grandes y significativas, como ocurre con: el alumnado se comporta ante una directora con igual respecto que ante un director (diferencia de 0.73 a favor de las directoras), si estoy descontenta con un trabajo, hablo con la persona afectada (0.73), afronto fácilmente aquellas situaciones en las que he de comunicar una mala noticia... (0.58) y alabo a mis colegas si considero que hacen un buen trabajo (0.52). Como se puede apreciar, la mayoría hacen referencia al ámbito del comportamiento asertivo y, hasta cierto punto, es normal que las 
directoras se vean más capaces en el afrontamiento de estas situaciones ya que, por su trabajo, han tenido mayores ocasiones de poner a prueba sus aptitudes y, mejorarlas, en los casos necesarios.

\section{Conclusiones}

A modo de síntesis, se destaca que las barreras que ambos grupos han señalado como más influyentes son la falta de políticas de apoyo a la maternidad y el conflicto entre los roles de trabajadora/cuidadora del hogar, mientras que otros aspectos como el acceso a la información y las actitudes hacia la gestión femenina no son considerados como negativos en el desarrollo profesional.

Nos llama la atención la diferencia en las auto-valoraciones de autoestima y asertividad a favor de las directoras. Otros trabajos (Carrasco, 2002; Díez Gutiérrez, 2004; García Gómez, 2006) han puesto de manifiesto una creencia en la falta de preparación para la dirección de la mujer frente al varón, justificada en el modelo de "mujer-madre-maestra", frente a la atribución a maestros y directores de mayor capacidad de control, orden, autoridad, etc. Podríamos pensar que es más fácil que impere este estereotipo en las docentes que no llegan a ser directoras, mientras que las que acceden al cargo sí han conseguido superarlo. Ello sin olvidar que el propio desempeño de la dirección puede contribuir a desarrollar un mayor sentimiento de valía.

No obstante, para ahondar en las diferencias entre directoras y maestras hubiera sido necesario analizar sus respuestas a la luz de otros datos profesionales y vitales (edad, número de hijos/as, edad del más pequeño/a, etc.). Así, podríamos comprobar si las docentes que llegan a directoras atraviesan por circunstancias parecidas o diferentes a las que no acceden al cargo, y de ahí sus diferentes opciones y opiniones.

Hay que destacar que tanto las docentes como las directoras encuestadas no consideran que ascender en el trabajo sea más importante que dedicar tiempo a la familia. El conflicto de roles, más que una barrera externa, es una consecuencia percibida de sus prioridades vitales. En el trabajo de Carrasco (2002) se pone de manifiesto que desempeñar la dirección es para muchas profesoras un tercer empleo, entendiéndose que iría en detrimento de los/as hijos/as.

Como reflexión personal ante estos datos, creemos importante cuestionar esta creencia de directoras y profesoras. Es como si prevaleciera la idea de que las profesoras prefieren ser madres a ser directoras; es decir, no se trata de que alguien les niegue el acceso a la dirección, sino que ellas mismas han optado por desarrollar y atender otras prioridades vitales. Es una elección personal y, por tanto, no se sienten excluidas ni discriminadas; en todo caso, se trataría de una barrera auto-impuesta.

Esta visión de la discriminación por razón de sexo es, si puede decirse así, un tanto inmadura, en tanto que todo lo que no sea una negación tajante, un obstáculo "visible", no es una discriminación (por eso insisten en que manejan la misma información que los varones, que están igual de capacitadas, etc.). Se "naturaliza" la discriminación; esta parece normal, fruto de elecciones y trayectorias personales voluntarias, obviándose los condicionamientos socioculturales. No llegan a cuestionarse el porqué son sólo las mujeres quienes han de optar entre la vida familiar y el ascenso profesional, o por qué sus preferencias profesionales 
son como son y no de otra manera y, por ello, estos hechos no se vislumbran como posibles indicios de desigualdades entre mujeres y hombres.

En este sentido, parece necesario potenciar un trabajo orientador con las docentes que les permita superar esta visión simplista de los obstáculos en su desarrollo profesional. Desde un punto de vista más general, habría incluso que plantear la necesidad de que los programas de orientación profesional para jóvenes, además de los contenidos tradicionales, incluyeran el análisis de los estereotipos de género que terminan limitando el proyecto de vida de las mujeres, sin que estas, en ocasiones, sean conscientes.

\section{Referencias Bibliográficas}

Ball, S. J. (1994). La micropolítica de la escuela. Hacia una teoría de la organización escolar. Barcelona. Paidós/MEC.

Bardisa, T. (1995). "La participación democrática en las instituciones educativas". En J. García y F. Delgado (Coords.), Los retos de la educación ante el siglo XXI (pp. 125-142). Madrid. Editorial Popular.

Carrasco, M. J. (2002). Análisis e incidencias de los estilos directivos de las mujeres en centros escolares de contextos desfavorecidos. Tesis doctoral. Universidad de Huelva.

Coronel, J. M., Moreno, E. y Padilla, M. T. (2002). "La gestión y el liderazgo como procesos organizativos: Contribuciones y retos planteados desde una óptica de género". Revista de Educación, $327,157-168$.

Diéz Gutiérrez, E. (dir.) (2004). La cultura de género en las organizaciones escolares: motivaciones y obstáculos de acceso de la mujer a los puestos de dirección. Madrid. Instituto de la Mujer. Consultado el 15 de enero de 2008 en: http://www.mtas.es/mujer/mujeres/estud_inves/630.pdf

Fitzgerald, L. F. y Weitzman, L. M. (1992). "Women's career development: theory and practice from a feminist perspective". En H. D. Lea y Z. B. Leibowitz, (Eds.), Adult career development (pp. 124-160). Alexandria, VA. The Nacional Career Development Association.

García Gómez, T. (2006). "La reproducción de estereotipos sexuales en las escuelas: un mecanismo de exclusión de las maestras del cargo de dirección". Bordón, 58 (1), 33-50.

Gimeno, M. J. y Rocabert, E. (1998). "Barreras percibidas por las mujeres en su proceso de toma de decisión vocacional”. Revista de orientación y psicología, 9 (15), 25-36.

Grañeras, M. (2003). "Las mujeres en los equipos directivos de los centros escolares en España". Organización y Gestión Educativa, 3, 15-20.

McWhirter, E. H., Torres, D. y Rasheed, S. (1998). "Assessing barriers to women's career adjustment". Journal of Career Assessment, 6 (4), 449-479.

Muñoz-Repiso, M. (2003). "Mujeres, educación y toma de decisiones en la Unión Europea”. Organización y Gestión Educativa, 3, 26-31.

Padilla, M. T. (2001). "Barreras y limitaciones en el desarrollo profesional de la mujer". Portularia. Revista de Trabajo Social, 1, 223-232.

Padilla, M. T. (2005). "Procedimiento para el análisis de la validez de contenido de una escala sobre condicionantes en el acceso a la dirección escolar". En Actas del XII Congreso Nacional de Modelos de Investigación Educativa: Investigación en Innovación Educativa (pp. 1196-1203). La Laguna. Servicio de Publicaciones de la Universidad de la Laguna.

Russell, J. E. A. y Burgess, J. R. D. (1998), "Success and women's career adjustment". Journal of Career Assessment, 6 (4), 365-387. 
Sánchez García, M. F. (2004). Orientación laboral. Para la diversidad y el cambio. Madrid. Sanz y Torres.

Suárez Ortega, M. (2004). Las mujeres adultas y los procesos de reinserción laboral. Un estudio en contextos locales de la provincia de Sevilla. Sevilla. Diputación de Sevilla.

Suárez Ortega, M. (2008). "Barreras en el desarrollo profesional femenino". Revista Española de Orientación y Psicopedagogía, 19 (1), 61-72.

Swanson, J. L. y Woitke M. B. (1997). "Theory into practice in career assessment for women: assessment and interventions regarding perceived barriers". Journal or Career Assessment, 5 (4), 443-462.

Fecha de recepción: 17-12-2007

Fecha de revisión: 20-01-2009

Fecha de aceptación: 18-02-2009 